\title{
Extracorporeal Irradiation in Malignant Bone Tumors
}

\author{
Ranjan Bhakta Bhandari,' Anjani Kumar Jha,' Pradeep Neupane,' Arun Sigdel,' Pradumna Prasad Chaurasia' \\ 'Department of radiation oncology, ${ }^{2}$ Department of Surgical Oncology, Orthopaedics Unit, BP Koirala Memorial Cancer \\ Hospital, Chitwan, Nepal.
}

\section{ABSTRACT}

Extracorporeal irradiation (ECI) is relatively a rare method used in the management of malignant bone tumors (MBT). It consists of en block removal of the tumor bearing bone segment, removal of the tumor from the bone, irradiation and re implantation back in the body.

Keywords: extracorporeal irradiation; malignant bone tumors; osteosarcoma.

\section{INTRODUCTION}

Primary malignant bone tumors (MBT) are relatively rare, but common in children and adolescents. Limb salvaging is most desirable mode of treatment. This can be possible by the use of multidisciplinary treatment which consists of optimal use of surgery, chemotherapy and radiotherapy. Extracapsular irradiation was first been reported in $1968 .^{1}$ The effected bone segment is removed from the body and irradiated and therefore avoidance of radiation exposure to the unirradiated bone muscles, joint and other healthy tissue of the body. High dose radiation is delivered (50G), lethal dose to remaining tumor cells and decreasing the chances of recurrence.

\section{CASE}

A $12 \mathrm{yr}$ old girl reported in Orthopedic OPD, with chief complains of painful swelling at her distal end of left thigh for two and half months. This was gradually increasing. Clinical examinations and pretreatment work up was done. The blood count was within normal limits, in biochemistry alk. phos was 352U/I,LDH 448u/l.

X-ray of It knee showed-mixed sclerotic and lytic mass lesion involving the metadiaphyseal aspect of the distal femur with soft tissue component, associated speculated periosteal reaction with codman triangle.
MRI left knee-Showed soft tissue component, appearing T1 isohypointense and T2 and FLAIR hyperintense arising from the metaphysical aspect of the distal femur. On contrast study, there was mild enhancement. There was also cortical erosion(esp lateral aspect) There was intra medullary increased signal on FLAIR and T2 sequence infiltration,infiltration to the muscular plane(esp vastus intermedius) as well as compressing the muscle(vastus medialis and lateralis) withedematos changes.The soft tissue was compressing the neurovascular bundle. Minimal increase fluid was noted in the medial compartment of the knee joint, $\mathrm{F} / \mathrm{S} / \mathrm{O}$ osteosarcoma left distal femur.

USG abdomen and pelvis-normal, CT chest-normal scan no features of metastasisNeedle biopsyOsteosarcoma

Patient underwent coraneal surgery, ${ }^{2,3}$ then the bone segment was tightly wrapped in wet sterile drape and then sealed in two plastic bags and was brought in radiotherapy department for $\mathrm{ECl}$.The wrapping material was thick enough to be sufficient for bolus effect in co-60. The bone segment was placed on the treatment

Correspondence: Dr. Ranjan Bhakta Bhandari, Department of radiation oncology, BP Koirala Memorial cancer Hospital Chitwan Nepal. Email: ranjanbhakła_bhandari@yahoo.com, Phone: +977. 9845152607. 
couch and immobility was ensured. The segment was irradiated with a single session dose of 50Gy prescribed at mid plane using C0-60.4 Two antero posterior fields was used. Radiation field size was chosen which adequately covered the segment, ${ }^{5,6,7}$ after the completion of $\mathrm{ECl}$ the bone segment was returned to operation theatre without delay. The time taken was less than 30 minutes from operation theatre to ECR procedure and back to operation theatre.

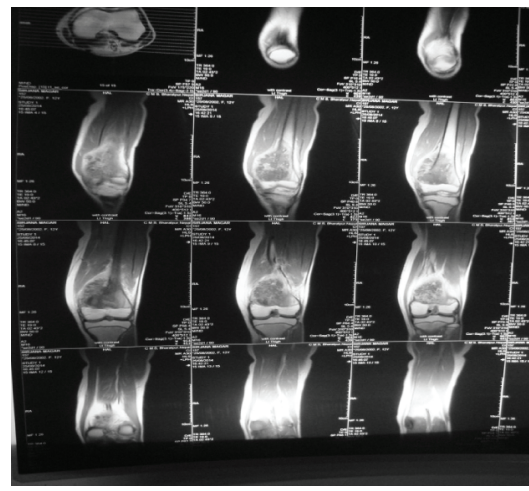

Clinical features

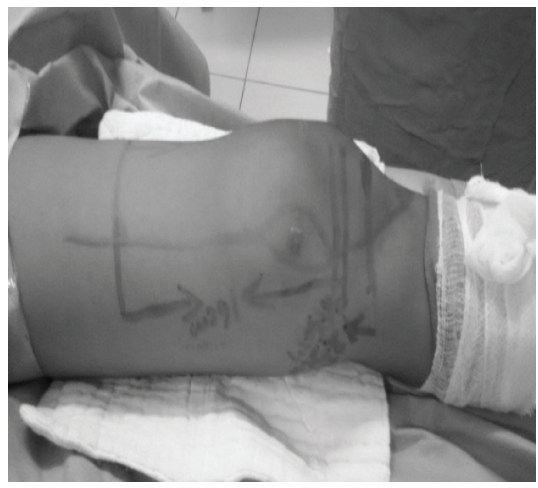

MRI

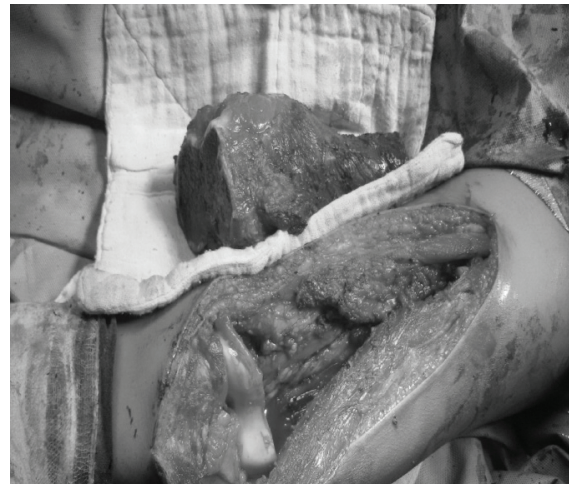

Per operative

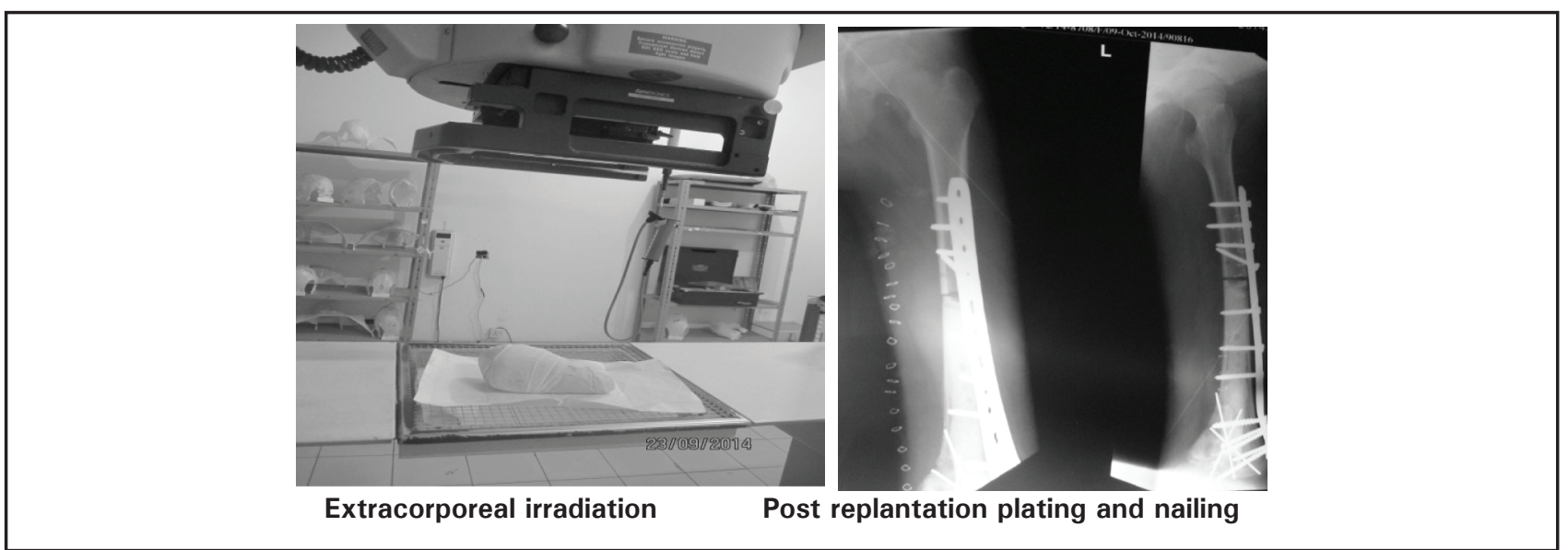

Post op period was uneventfull, wound healed well, patient is recovering well, ${ }^{8}$ no complications are noted.

\section{DISCUSSION}

Organ preservation is most important in the treatment of malignant bone tumors. Extracorporeal irradiation provides an option in limb salvaging in osteosarcoma and other malignant bone tumors. Though it has been reported only in three cases in BP Koirala Memorial cancer hospital, the experience gained and the confidence built with these cases in future further cases can also be tried for extracorporeal irradiation and Limb salvaging, organ preservation can be achieved in coming days.

\section{REFERENCES}

1. Spira E, Lubin E. Extracorporeal irradiation of bone tumors .A preliminary report. Isr J Med sci 1968;4:1015-9.

2. Araki N, Myoui A, Kuratsu S, Hashimoto N, Inoue T, Kudwara I, et al. Intraoperative extracorporeal autogenius irradiated bone graft in tumor surgery.Clin Orthop Relat Res 1999 Nov;368:196-206.
3. Mankin HJ, Gebhardt MC, Jenning LC, Springfield DS, Tomford WW. Long terms results of allograft replacement in the management of bone tumors.Clinc. Orthop 1996:324:86-97.

4. Ajaya Puri, Ashis Gulia, MG Agarwal et al. Extracorporeal irradiated tumor bone: A reconstruction option in diaphyseal 
Ewings sarcoma.Indian J orthop, 2010 oct-Dec; 44(4):390-396.

5. Honga A, Stevens G, Stalley P, et al. Extracorporeal irradiation for malignant bone tumors. Int Radiat oncol Biol Phys 2001;50:441-7.

6. Sabo D, Bernd $\mathrm{L}$, Buchner $\mathrm{M}$ et al. Intraoperative extracorporeal irradiation and replantation of primary malignant bone tumors. Orthopade 2003;32:1003-12.
7. Chen WM, Huang CK, Chiang CC, Low H. Treatment of malignant bone tumors by extracorporeal irradiated autograft-prosthetic composite anthroplasty. $\mathrm{T}$ Bone joint $\operatorname{surg}(\mathrm{Br}) 2002 ; 84-\mathrm{B}: 1156-61$.

8. Currey JD, Foreman J, Laktic I et al. Effects of ionizing radiation on the mechanical properties of human bone J. orthop Res 1997;15-111-17. 